\title{
Macitentan attenuates chronic mountain sickness in rats by regulating arginine and purine metabolism
}

Xiaojian $\mathrm{Gao}^{1}$, Zeyu Zhang ${ }^{1}$, Xin $\mathrm{Li}^{2,3}$, Chen $\mathrm{Li}^{2,3}$, Jianxiu $\mathrm{Hao}^{2,3}$, Yunfu Luo ${ }^{4}$, Maoyi $\mathrm{Lei}^{4}$, Junmiao $\mathrm{Li}^{4}$, Chunlei $\mathrm{Liu}^{2,3 *}$, Kunlun $\mathrm{He}^{2,3 * *}$

${ }^{1}$ Department of Cardiovascular, Chinese PLA General Hospital, Beijing 100853, China ${ }^{2}$ Laboratory of Translational Medicine, Chinese PLA General Hospital, Beijing, China

${ }^{3}$ Beijing key laboratory of chronic heart failure precision medicine, Chinese PLA General Hospital, Beijing, China

4 WuXi AppTec Co., Ltd., 288 Fute Zhong Road, Waigaoqiao Free Trade Zone, Shanghai 200131, China $^{1}$

contents:

Page Title

Fig. S1 S2 Pearson's correlation heatmap of metabolites and mPAP

Fig. S2 S2 Representative images of the entire membrane in WB experiment

Table S1 S3 Correlation analysis between metabolites and mPAP 


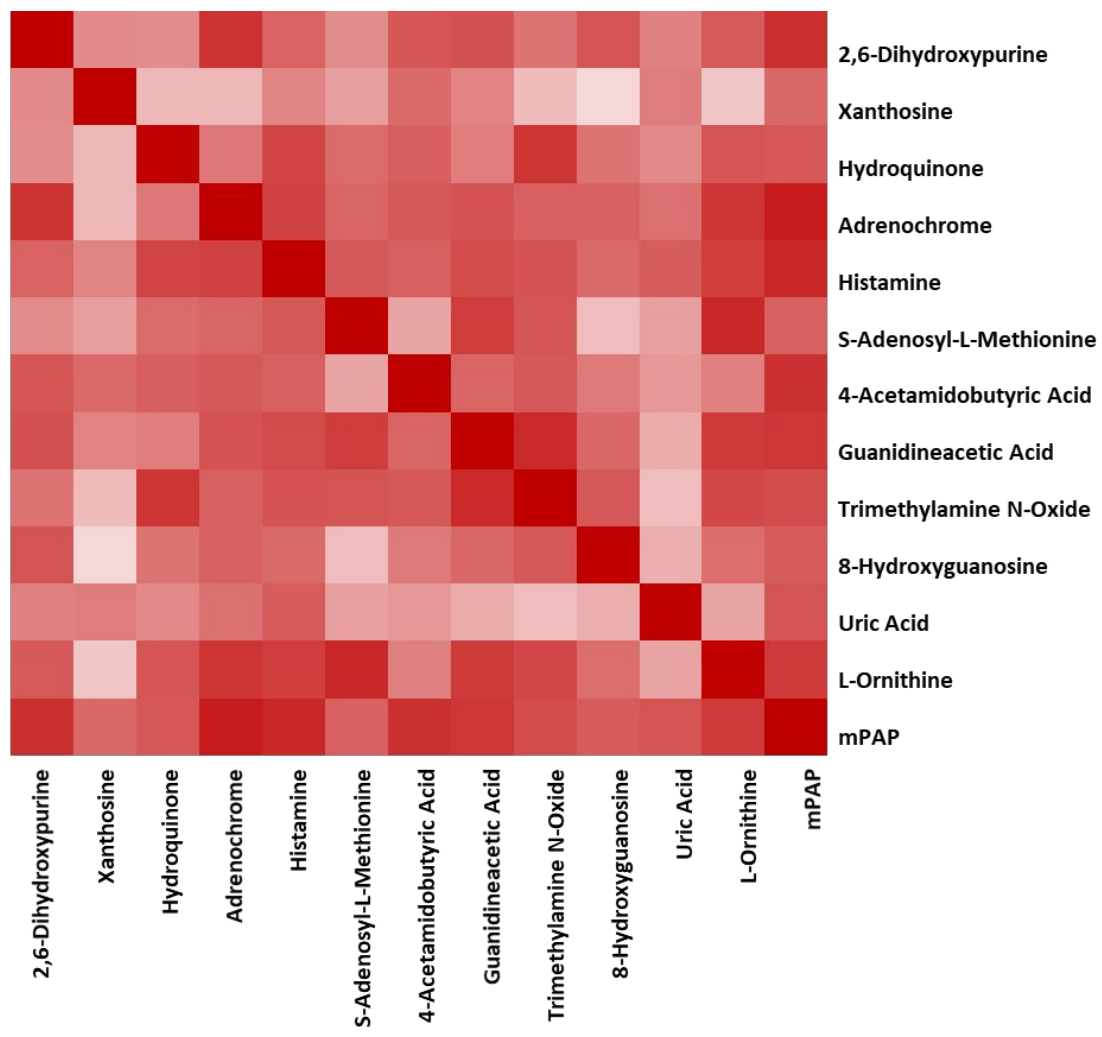

Fig. S1. Pearson's correlation heatmap of metabolites and mPAP
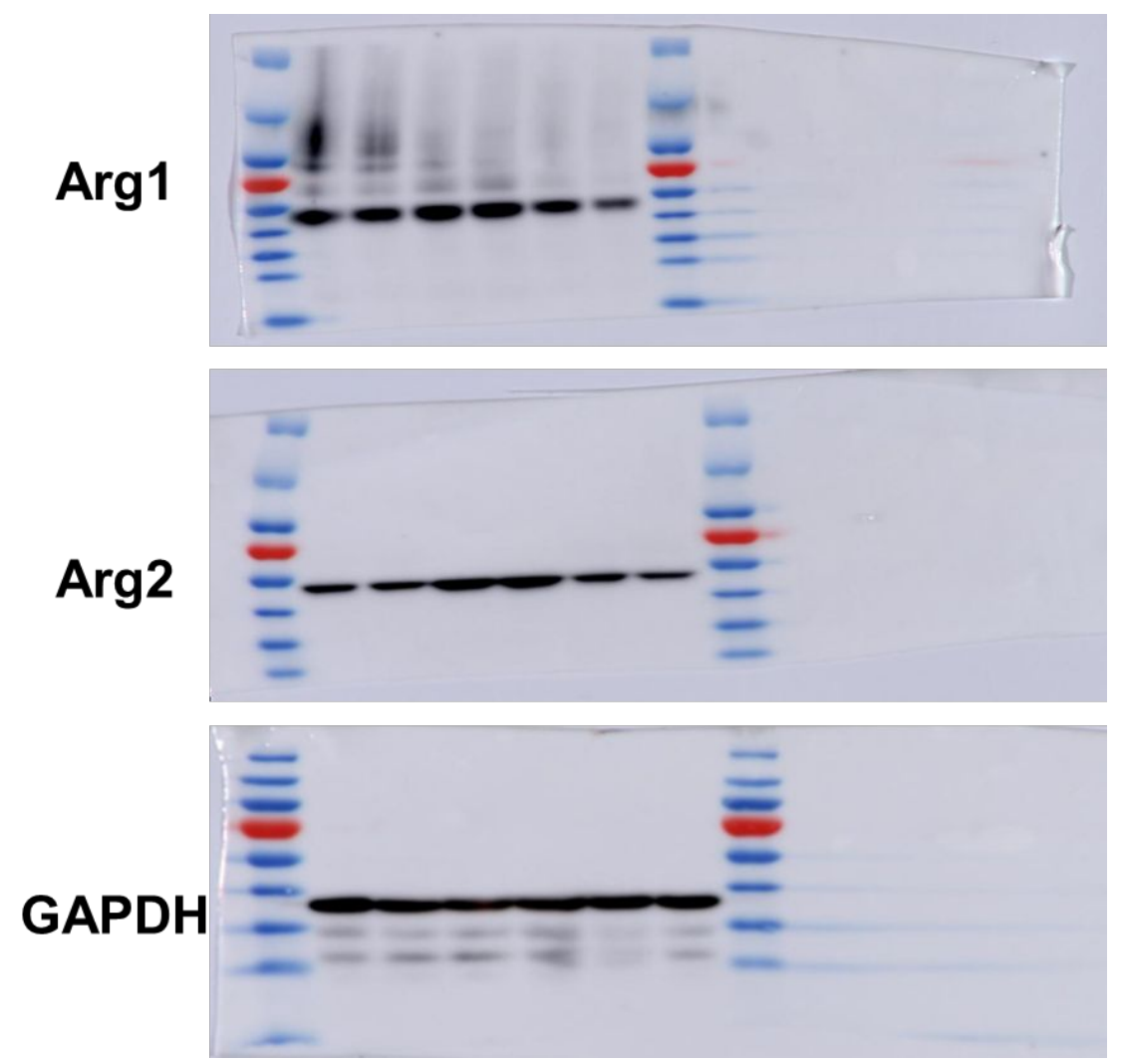

Fig. S2. Representative images of the entire membrane in WB experiment 
Table S1. Correlation analysis between metabolites and mPAP

\begin{tabular}{|c|c|c|c|c|c|c|c|c|c|c|c|c|c|c|}
\hline & & $\begin{array}{c}\text { 2,6-Dihydroxy } \\
\text { purine }\end{array}$ & Xanthosine & $\begin{array}{c}\text { Hydro } \\
\text { quinone }\end{array}$ & $\begin{array}{l}\text { Adreno } \\
\text { chrome }\end{array}$ & Histamine & $\begin{array}{l}\text { S-Adenosyl- } \\
\text { IMethionine }\end{array}$ & $\begin{array}{c}\text { 4-Acetamido } \\
\text { butyricAcid }\end{array}$ & Guanidineacetic & $\begin{array}{c}\text { Trimethylamine } \\
\text { NOxide }\end{array}$ & $\begin{array}{l}\text { 8-Hydroxy } \\
\text { guanosine }\end{array}$ & UricAcid & L-Ornithine & mPAP \\
\hline \multirow{2}{*}{$\begin{array}{c}2,6- \\
\text { Dihydroxypurine }\end{array}$} & $\mathrm{r}$ & 1 & .460 & .450 & $.804^{* *}$ & $.614^{*}$ & .452 & $.664^{* *}$ & $.684^{* * *}$ & $.552^{*}$ & $.674^{* *}$ & .492 & $.649^{* *}$ & $.823^{* *}$ \\
\hline & $P$ & - & .084 & .092 & .000 & .015 & .091 & .007 & .005 & .033 & .006 & .062 & .009 & .000 \\
\hline \multirow[t]{2}{*}{ Xanthosine } & $\mathrm{r}$ & .460 & 1 & .280 & .285 & .483 & .382 & $.583^{*}$ & .487 & .269 & .158 & .510 & .224 & $.583^{*}$ \\
\hline & $P$ & .084 & - & .312 & .302 & .068 & .160 & .023 & .066 & .332 & .574 & .052 & .422 & .023 \\
\hline \multirow[t]{2}{*}{ Hydroquinone } & $\mathrm{r}$ & .450 & .280 & 1 & $.535^{*}$ & $.732^{* *}$ & $.578^{*}$ & $.629^{*}$ & .506 & $.792^{* *}$ & $.551^{*}$ & .465 & $.664^{* *}$ & $.651^{* *}$ \\
\hline & $P$ & .092 & .312 & - & .040 & .002 & .024 & .012 & .054 & .000 & .033 & .081 & .007 & .009 \\
\hline \multirow[t]{2}{*}{ Adrenochrome } & $\mathrm{r}$ & $.804^{* *}$ & .285 & $.535^{*}$ & 1 & $.746^{* *}$ & $.607^{*}$ & $.652^{* *}$ & $.676^{* *}$ & $.627^{*}$ & $.622^{*}$ & $.561^{*}$ & $.790^{* *}$ & $.892^{* *}$ \\
\hline & $P$ & .000 & .302 & .040 & - & .001 & .016 & .008 & .006 & .012 & .013 & .030 & .000 & .000 \\
\hline \multirow[t]{2}{*}{ Histamine } & $\mathrm{r}$ & $.614^{*}$ & .483 & $.732^{* *}$ & $.746^{* *}$ & 1 & $.654^{* *}$ & $.627^{*}$ & $.704^{* *}$ & $.676^{* *}$ & $.589^{*}$ & $.636^{*}$ & $.758^{* *}$ & $.844^{* *}$ \\
\hline & $P$ & .015 & .068 & .002 & .001 & - & .008 & .012 & .003 & .006 & .021 & .011 & .001 & .000 \\
\hline S_Adenosyl & $\mathrm{r}$ & .452 & .382 & $.578^{*}$ & $.607^{*}$ & $.654^{* *}$ & 1 & .355 & $.761^{* *}$ & $.673^{* *}$ & .260 & .380 & $.845^{* *}$ & $.612^{*}$ \\
\hline LMethionine & $P$ & .091 & .160 & .024 & .016 & .008 & - & .194 & .001 & .006 & .350 & .162 & .000 & .015 \\
\hline \multirow{2}{*}{$\begin{array}{l}\text { 4-Acetamidobu } \\
\text { tyric Acid }\end{array}$} & $\mathrm{r}$ & $.664^{* *}$ & $.583^{*}$ & $.629^{*}$ & $.652^{* *}$ & $.627^{*}$ & .355 & 1 & $.606^{*}$ & $.653^{* *}$ & $.524^{*}$ & .407 & .495 & $.792^{* *}$ \\
\hline & $P$ & .007 & .023 & .012 & .008 & .012 & .194 & - & .017 & .008 & .045 & .132 & .060 & .000 \\
\hline \multirow{2}{*}{$\begin{array}{l}\text { Guanidineacetic } \\
\text { Acid }\end{array}$} & $\mathrm{r}$ & $.684^{* *}$ & .487 & .506 & $.676^{* *}$ & $.704^{* *}$ & $.761^{* *}$ & $.606^{*}$ & 1 & $.836^{* *}$ & $.593^{*}$ & .329 & $.776^{* *}$ & $.793^{* *}$ \\
\hline & $P$ & .005 & .066 & .054 & .006 & .003 & .001 & .017 & - & .000 & .020 & .231 & .001 & .000 \\
\hline \multirow{2}{*}{$\begin{array}{l}\text { Trimethylamine } \\
\text { NOxide }\end{array}$} & $\mathrm{r}$ & $.552^{*}$ & .269 & $.792^{* *}$ & $.627^{*}$ & $.676^{* *}$ & $.673^{* *}$ & $.653^{* *}$ & $.836^{* *}$ & 1 & $.652^{* *}$ & .261 & $.724^{* *}$ & $.703^{* *}$ \\
\hline & $P$ & .033 & .332 & .000 & .012 & .006 & .006 & .008 & .000 & - & .008 & .348 & .002 & .003 \\
\hline \multirow{2}{*}{$\begin{array}{l}\text { 8-Hydroxy } \\
\text { guanosine }\end{array}$} & $\mathrm{r}$ & $.674^{* *}$ & .158 & $.551^{*}$ & $.622^{*}$ & $.589^{*}$ & .260 & $.524^{*}$ & $.593^{*}$ & $.652^{* *}$ & 1 & .315 & $.569^{*}$ & $.667^{* *}$ \\
\hline & $P$ & .006 & .574 & .033 & .013 & .021 & .350 & .045 & .020 & .008 & - & .253 & .027 & .007 \\
\hline \multirow[t]{2}{*}{ UricAcid } & $\mathrm{r}$ & .492 & .510 & .465 & $.561^{*}$ & $.636^{*}$ & .380 & .407 & .329 & .261 & .315 & 1 & .359 & $.663^{* *}$ \\
\hline & $P$ & .062 & .052 & .081 & .030 & .011 & .162 & .132 & .231 & .348 & .253 & - & .188 & .007 \\
\hline \multirow[t]{2}{*}{ L-Ornithine } & $\mathrm{r}$ & $.649^{* *}$ & .224 & $.664^{* *}$ & $.790^{* *}$ & $.758^{* *}$ & $.845^{* *}$ & .495 & $.776^{* *}$ & $.724^{* *}$ & $.569^{*}$ & .359 & 1 & $.776^{* *}$ \\
\hline & $P$ & .009 & .422 & .007 & .000 & .001 & .000 & .060 & .001 & .002 & .027 & .188 & - & .001 \\
\hline \multirow[t]{2}{*}{ mPAP } & $r$ & $.823^{* *}$ & $.583^{*}$ & $.651^{* *}$ & $.892^{* *}$ & $.844^{* *}$ & $.612^{*}$ & $.792^{* *}$ & $.793^{* *}$ & $.703^{* *}$ & $.667^{* *}$ & $.663^{* *}$ & $.776^{* *}$ & 1 \\
\hline & $P$ & .000 & .023 & .009 & .000 & .000 & .015 & .000 & .000 & .003 & .007 & .007 & .001 & - \\
\hline
\end{tabular}


mPAP :mean pulmonary arterial pressure, r: Pearson's correlation coefficient, $* * P<0.01, * P<0.05$ 